\title{
Show Me the Evidence
}

\author{
Wendy C. Budin, PhD, RN-BC, FAAN, LCCE, FACCE
}

\begin{abstract}
In this column, the editor of The Journal of Perinatal Education discusses the need for current high-quality evidence to guide clinical practice. The editor also describes the contents of this issue, which offer a broad range of resources, research, and inspiration for childbirth educators in their efforts to promote, support, and protect natural, safe, and healthy birth.
\end{abstract}

The Journal of Perinatal Education, 25(4), 203-204, http://dx.doi.org/10.1891/1058-1243.25.4.203

Keywords: Toolkit, primary cesareans, evidence-based practice, normal birth, natural birth, safe birth, healthy birth, physiological childbirth education, perinatal education, breastfeeding

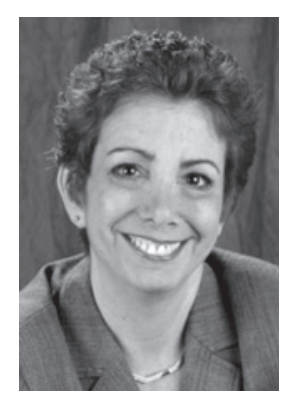

Wendy C. Budin Editor
I recently read an article reporting the results of the first randomized controlled trial (RCT) in Australia that investigated the effectiveness of a birth preparation course, integrating multiple complementary medicine (CM) techniques, for the support of natural birth for first-time mothers (Levett, Smith, Bensoussan, \& Dahlen, 2016). The authors demonstrated that antenatal education classes focusing on pain relief techniques dramatically reduce the rate of medical interventions during childbirth, such as epidural use and cesarean surgery. What makes the results of this study noteworthy is that the authors were able to conduct a well-designed RCT. It is rare that we see RCTs conducted on the effectiveness of childbirth education. RCTs have within them, by the nature of randomization, an ability to help control bias. Bias can confound the outcome of a study such that the study may over- or underestimate what the true treatment effect is.

When applying new evidence to practice, that is, implementing evidence-based practice, it requires the integration of clinical judgment, patient preferences and values, and recommendations from the best available evidence. To identify the "best available evidence," clinicians or consumers of research need to have a clear understanding of appraisal skills to judge the study strengths and also knowledge of the hierarchy or levels of evidence to decide if and when new evidence can be used to change practice. According to most evidence pyramids or hierarchies, systematic reviews such as findings from a Cochrane review are at the pinnacle or top of the pyramid as the strongest form of evidence. Systematic reviews synthesize the results of multiple RCTs. The next strongest level of evidence, right below systematic review on the evidence hierarchy, is a single well-designed RCT.

When applying new evidence to practice, that is, implementing evidence-based practice, it requires the integration of clinical judgment, patient preferences and values, and recommendations from the best available evidence. 
The content of all JPE issues published since October 1998 is available on the journal's website (http:// www.ingentaconnect. com/content/springer/ jpe). Lamaze International members can access the site and download free copies of JPE articles by logging on at the "Members Only" link on the Lamaze website (http:// www.lamaze.org)
Thus, the study of Levett et al. (2016) would provide a high level of evidence to support the use of birth preparation focusing on complementary pain relief techniques to reduce the rate of medical interventions during childbirth, such as epidural use and cesarean surgery. These findings strengthen the current available evidence of the supportive role from complementary pain management techniques and may offer women additional options for managing pain in labor. As the authors suggest, however, further confirmation of these findings is recommended.

\section{IN THIS ISSUE}

In this issue of The Journal of Perinatal Education feature article, Debby Amis describes the role of the childbirth educator in supporting vaginal birth and reducing primary cesareans. She presents highlights from the California Maternal Quality Care Collaborative evidence-based, comprehensive Toolkit to support vaginal birth and reduce primary cesareans. This article highlights the recommended strategies from the "Toolkit" of particular interest to childbirth educators.

In this issue's "Celebrate Birth!" column, Megan Pagano, a new mother eloquently describes the intense pain and joy she experienced while effectively coping with an intense and challenging 5-hour labor with her first child.

Turning to practice, Renece Waller-Wise discusses how a birth plan can be used as a tool for promoting patient engagement. When patients have an active role in their health care, childbirth educators and nurses are in a position to support patient choices. Waller-Wise suggests that when patients are engaged and stay engaged, the results can be improved patient care outcomes and improved patient satisfaction. This process can be facilitated through education of choices and assisting with writing choices into a formal birth plan.

Canadian authors Young, Twells, Joy, Newhook, Goodridge, and Burrage present the finding of their study that examined primary factors that influenced grandmothers' choices of infant feeding and explored the role that grandmothers felt they played in their daughters' choices about infant feeding. Twenty-two maternal grandmothers who bottle fed their children and whose daughters also bottle fed their babies were recruited to participate in four focus groups and/or two interviews. Three themes emerged that described how grandmothers felt about their infant feeding experiences: "Powerlessness," "Modesty," and "Ambivalence." These themes and their implications are discussed in this article.

Also in this issue we present findings from a study by Kovala, Cramp, and Xia exploring expectant parents' perception of importance regarding a wide range of prenatal education topics and their information delivery method preferences. Authors identified the topics of newborn safety, birth, and breastfeeding as most important to participants. Most of the participants also indicated that their preference was to attend face-to-face-prenatal education sessions. Findings from this study provide valuable information to inform future prenatal education program content and delivery.

Finally, we share an important contribution to the literature about gestational weight gain (GWG). Champlin, Walker, and Mackert share the results from their innovative scoping review of GWG through a health literacy lens. These authors show few women gain the recommended amount of weight during pregnancy, which has health implications for mothers and their newborns. The purpose of this project was to review literature on a more patient-centered concept—health literacy. A scoping review was conducted to determine whether aspects of health literacy are included in GWG research. Although studies included health literacy aspects indirectly, only two directly measured health literacy using existing measures. The authors conclude that health literacy may be a critical, yet understudied, factor in understanding why GWG falls outside of the recommendations.

\section{REFERENCE}

Levett, K. M., Smith, C. A., Bensoussan, A., \& Dahlen, H. G. (2016). Complementary therapies for labour and birth study: A randomised controlled trial of antenatal integrative medicine for pain management in labour. BMJ Open, 6, e010691. http://dx.doi.org/10.1136/bmjopen2015-010691

WENDY C. BUDIN is the editor-in-chief of The Journal of Perinatal Education. She is also the research scientist and adjunct professor at New York University, College of Nursing. She is a fellow in the American College of Childbirth Educators and is chair of the Lamaze International Certification Council. 